\title{
Probing photoelectron angular distributions in molecules with polarization-controlled two-color above-threshold ionization
}

\author{
Torsten Leitner, ${ }^{1, *}$ Richard Taïeb, ${ }^{2,3}$ Michael Meyer, ${ }^{4}$ and Philippe Wernet ${ }^{1, \dagger}$ \\ ${ }^{1}$ Institute for Methods and Instrumentation for Synchrotron Radiation Research, Helmholtz-Zentrum Berlin für Materialien und Energie \\ GmbH, Albert-Einstein-Strasse 15, 12489 Berlin, Germany \\ ${ }^{2}$ Sorbonne Université, UPMC, Université Paris 06, Laboratoire de Chimie Physique-Matière et Rayonnement, UMR 7614, \\ 11 Rue Pierre et Marie Curie, 75231 Paris Cedex 05, France \\ ${ }^{3}$ CNRS, LCPMR, UMR 7614, Paris Cedex 05, France \\ ${ }^{4}$ European XFEL GmbH, Albert-Einstein-Ring 19, 22761 Hamburg, Germany
}

(Received 5 January 2015; published 17 June 2015)

\begin{abstract}
We present polarization-controlled multiphoton two-color above-threshold ionization (TCATI) of molecules. The intensity modulations of valence photoelectron intensities of molecules arising from varying the relative orientation of the linear polarization vectors of femtosecond infrared (IR) and vacuum-ultraviolet (VUV) radiation in TCATI of the highest occupied molecular orbitals of $\mathrm{H}_{2} \mathrm{O}, \mathrm{O}_{2}$, and $\mathrm{N}_{2}$ are reported. The results on the molecular systems are compared to the $3 p$ photoionization of atomic Ar, which serves as a reference system. Modeling the large differences of the modulation amplitudes within the soft-photon approximation enables us to extract the one-photon-ionization anisotropy parameter $\beta_{2}$. Accounting only for the first sideband due to two-photon TCATI by one VUV and one IR photon we find satisfactory agreement between experiment and simulation for $\mathrm{H}_{2} \mathrm{O}$ and $\mathrm{O}_{2}$. However, the model fails for $\mathrm{N}_{2}$ and possible reasons are discussed. We discuss that the described approach may represent an alternative way of determining photoelectron angular distributions from valence shells of molecules and indicate future directions for modeling TCATI of molecules.
\end{abstract}

PACS number(s): $32.80 . \mathrm{Fb}, 32.80 . \mathrm{Rm}$

The occurrence of sidebands in atomic photoemission with femtosecond vacuum-ultraviolet (VUV) pulses in the presence of an infrared (IR) dressing field was already discovered more than 20 years ago for the laser-assisted Auger decay (LAAD) [1] and for two-color above-threshold ionization (TCATI) [2]. Sidebands occur when the photon density of the dressing field is high enough to enable simultaneous absorption or emission of one or more IR photons by the photoelectron arising from ionization with the VUV pulse [3,4]. They are expressed in the spectra as satellite lines shifted in kinetic energy with respect to the main line from single-photon VUV ionization by the energy of one or more IR photons. As the sideband intensity represents a cross-correlation signal of the femtosecond IR and VUV pulses, two-photon TCATI on gaseous as well as solid samples has ever since been used to determine the temporal resolution in pump-probe photoelectron spectroscopy experiments with laboratory VUV and soft X-ray sources and free-electron lasers (FELs) [5-17].

In TCATI in atoms it was discovered that the sideband intensity depends on the relative orientations of the linear polarization vectors of IR and VUV radiation [18,19]. This effect was then used in polarization-controlled TCATI to extract the angular distribution and the symmetry of the outgoing TCATI electron waves in $\mathrm{He}$ as a function of the dressing photon field density [20]. The polarization dependence in TCATI can be understood in terms of polarization-dependent coupling of the intermediate states with the continua, and the angular distribution is in general described by two asymmetry parameters, $\beta_{2}$ and $\beta_{4}[21,22]$.

\footnotetext{
*Present address: Dept. of Physics and Astronomy, Uppsala University, PO Box 516, 75120 Uppsala, Sweden.

${ }^{\dagger}$ wernet@helmholtz-berlin.de
}

An atomic model connecting sideband polarization sensitivity with the one-photon-ionization anisotropy parameter $\beta_{2}$, which characterizes the photoelectron angular distributions, was introduced in [18]. An alternative to the approach of varying the relative polarizations of IR and VUV radiation by angular detection of the photoelectrons was established for $\mathrm{He}$ and Ar in Refs. [23-29]. This enabled studying the dependence of the TCATI electron angular distributions on their kinetic energy and on the photon energy of the dressing field [26], and it was used to determine the polarization state of a VUV free-electron laser source [28]. The TCATI sideband photoelectrons were furthermore predicted to exhibit dichroism effects for circularly polarized IR and VUV radiation [30,31] and nondipole effects in the angular distribution [32]. Finally, polarization-controlled TCATI was used to determine the symmetry of the outgoing TCATI electron waves in the sequential two-photon double ionization in $\mathrm{Ne}^{+}$[33].

Investigations on TCATI in molecules and particularly on the polarization dependence of TCATI in molecules are needed. The question arises whether the polarization dependence of TCATI is sensitive to the molecular symmetry. Can we "turn the tables" on polarization-controlled TCATI as it has been applied so far and use the polarization dependence of the TCATI sideband electrons to extract information on the angular distribution of the main photoelectron lines in molecules? This could add an approach to the suite of methods for measuring the angular distribution of photoelectrons in molecules [34] by angular-resolved detection of the electrons with two or more electron energy analyzers [34-39], by imaging the electron trajectories with, e.g., velocity map imaging [23-29], by aligning or orienting the target in space [40-45], or by performing the measurement in the molecular frame by correlating ion and electron momenta with, e.g., coincidence techniques [46-48]. 

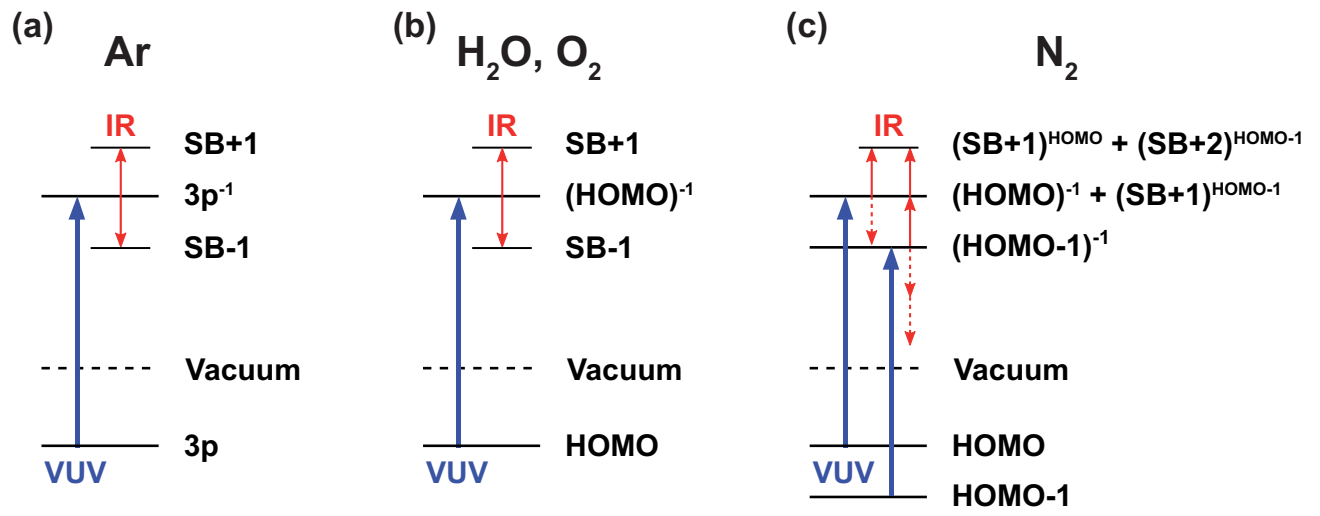

FIG. 1. (Color online) Schematic depiction of two-color above-threshold ionization with the essential processes observed in experiment for (a) Ar $3 p$ photoionization, (b) photoionization of the HOMO in $\mathrm{H}_{2} \mathrm{O}$ and $\mathrm{O}_{2}$, and (c) photoionization of $\mathrm{HOMO}$ and $\mathrm{HOMO}-1$ in $\mathrm{N}_{2}$. In this one-electron picture the orbital energies are plotted with respect to the common vacuum level. SB stands for sideband.

Here we present our combined experimental and theoretical investigation of polarization-controlled TCATI in gaseous $\mathrm{H}_{2} \mathrm{O}, \mathrm{O}_{2}$, and $\mathrm{N}_{2}$. At the comparably low dressing-field intensity used here only one sideband on each side of the main photoelectron line is observed. Comparing the results for the three molecules, large differences in the sidebandintensity modulation amplitudes are found when varying the relative orientation between the dressing-field IR and the VUV polarization vectors. The results are analyzed with respect to the $3 p$ photoionization of atomic Ar gas serving as a reference case. An atomic model based on the soft-photon approximation and accounting for the first sideband from two-photon TCATI with one VUV and one IR photon enables us to extract the one-photon-ionization anisotropy parameter $\beta_{2}$. We thus demonstrate a first step towards determining photoelectron angular distributions in molecules with polarization-controlled TCATI.

The experiment was carried out with the setup described in detail in [10]. The 15th harmonic (photon energy $23.7 \mathrm{eV}$ ) was overlapped in time and space with the fundamental of an intense $(0.25 \mathrm{~mJ})$ IR laser $(785 \mathrm{~nm}, 1.58 \mathrm{eV})$. The VUV radiation was used to ionize the gaseous targets and the superimposed IR pulses were creating a dressing field of about $10^{12} \mathrm{~W} / \mathrm{cm}^{2}$ in the source volume of an angle-integrating magnetic-bottle-type electron energy analyzer. The temporal overlap was achieved by maximizing the sideband intensity for $\mathrm{Ar}$ ionization for varying time delay between IR and VUV pulses. The pulse durations of IR and VUV pulses amounted to 60 and $120 \mathrm{fs}$, respectively. The relative polarization of the two pulses was varied by stepwise rotating the polarization axis of the IR radiation using a rotatable half-wave plate, and photoelectron spectra were measured for various relative orientations. The IR dressing-field photon density was limited so that TCATI was dominated by contributions where, simultaneously to VUV ionization, only one additional IR photon is absorbed or emitted with only one sideband on each side of the main line. This is schematically depicted for Ar in Fig. 1(a) and for $\mathrm{H}_{2} \mathrm{O}$ and $\mathrm{O}_{2}$ in Fig. 1(b). The more complicated scheme for $\mathrm{N}_{2}$ in Fig. 1(c) will be discussed later.

For the present study, we investigate valence photoionization of the molecular targets $\mathrm{H}_{2} \mathrm{O}, \mathrm{O}_{2}$, and $\mathrm{N}_{2}$. We restrict ourselves to ionization of the highest occupied molecular orbitals (HOMOs) as the relating first sideband from twophoton TCATI with one VUV plus one IR photon can be best separated from intensities in the spectrum relating to other processes such as higher-order sidebands from deeper-lying valence orbitals. The initial ground- and the final ionic-state electronic configurations for one-photon VUV ionization of the HOMO are given in Table I for $\mathrm{H}_{2} \mathrm{O}, \mathrm{O}_{2}$, and $\mathrm{N}_{2}$ and for $3 p$ ionization of Ar.

The valence photoelectron spectra of $\mathrm{Ar}, \mathrm{H}_{2} \mathrm{O}, \mathrm{O}_{2}$, and $\mathrm{N}_{2}$ including the polarization-controlled TCATI signals are depicted in Fig. 2 for parallel and perpendicular polarization of dressing IR and VUV fields. The spectra were calibrated to the tabulated binding energies and reflect the well-known valence electronic structure of the systems [49]. The main lines in the spectra arise from one-photon VUV ionization of the HOMOs for the molecules and of the $3 p$ valence shell for $\mathrm{Ar}$ (see Table I). The first sidebands are clearly visible at $1.58 \mathrm{eV}$ higher and lower binding energies of the main lines. Second-order sidebands resulting from the interaction with two IR photons and separated by $3.2 \mathrm{eV}$ from the main line are present, but much weaker in intensity. The following analysis is limited to the first sidebands at lower binding energies of the main lines ("SB+1" in Figs. 1 and 2) and the contributions from second-order sidebands are neglected for the moment. As can be readily seen in Fig. 2, the modulations of the sideband intensities with the relative polarizations of IR and VUV radiation are strongly different for the different systems. For $\mathrm{Ar}$ and $\mathrm{H}_{2} \mathrm{O}$ the intensity drops by a factor of 2 when changing the polarization vectors from parallel to perpendicular orientations. In contrast, the variations appear to be particularly small for $\mathrm{O}_{2}$ and $\mathrm{N}_{2}$. In order to quantify

TABLE I. Initial ground- and final ionic-state electronic configurations for one-photon VUV ionization for $3 p$ photoionization of $\mathrm{Ar}$ and for photoionization of the $\mathrm{HOMO}$ of $\mathrm{H}_{2} \mathrm{O}, \mathrm{O}_{2}$, and $\mathrm{N}_{2}$.

\begin{tabular}{llll}
\hline \hline \multicolumn{2}{c}{ Initial ground state } & \multicolumn{3}{c}{ Final ionic state } \\
\hline $\mathbf{A r}$ & $3 s^{2} 3 p^{6}\left({ }^{1} S_{0}\right)$ & $\mathbf{A r}^{+}$ & $3 s^{2} 3 p^{5}\left({ }^{2} P_{1 / 2,3 / 2}\right)$ \\
$\mathbf{H}_{2} \mathbf{O}$ & $\left(1 b_{1} 2 p\right)^{2}\left({ }^{1} A_{1}\right)$ & $\mathbf{H}_{\mathbf{2}} \mathbf{O}^{+}$ & $\left(1 b_{1} 2 p\right)^{1}\left({ }^{2} B_{1}\right)$ \\
$\mathbf{O}_{2}$ & $\left(\pi_{g}^{*} 2 p\right)^{2}\left({ }^{3} \Sigma_{g}^{-}\right)$ & $\mathbf{O}_{2}^{+}$ & $\left(\pi_{g}^{*} 2 p\right)^{1}\left({ }^{2} \Pi_{g}\right)$ \\
$\mathbf{N}_{\mathbf{2}}$ & $\left(\sigma_{\mathrm{g}} 2 p\right)^{2}\left({ }^{1} \Sigma_{g}^{+}\right)$ & $\mathbf{N}_{\mathbf{2}}^{+}$ & $\left(\sigma_{\mathrm{g}} 2 p\right)^{1}\left({ }^{2} \Sigma_{g}^{+}\right)$ \\
\hline \hline
\end{tabular}




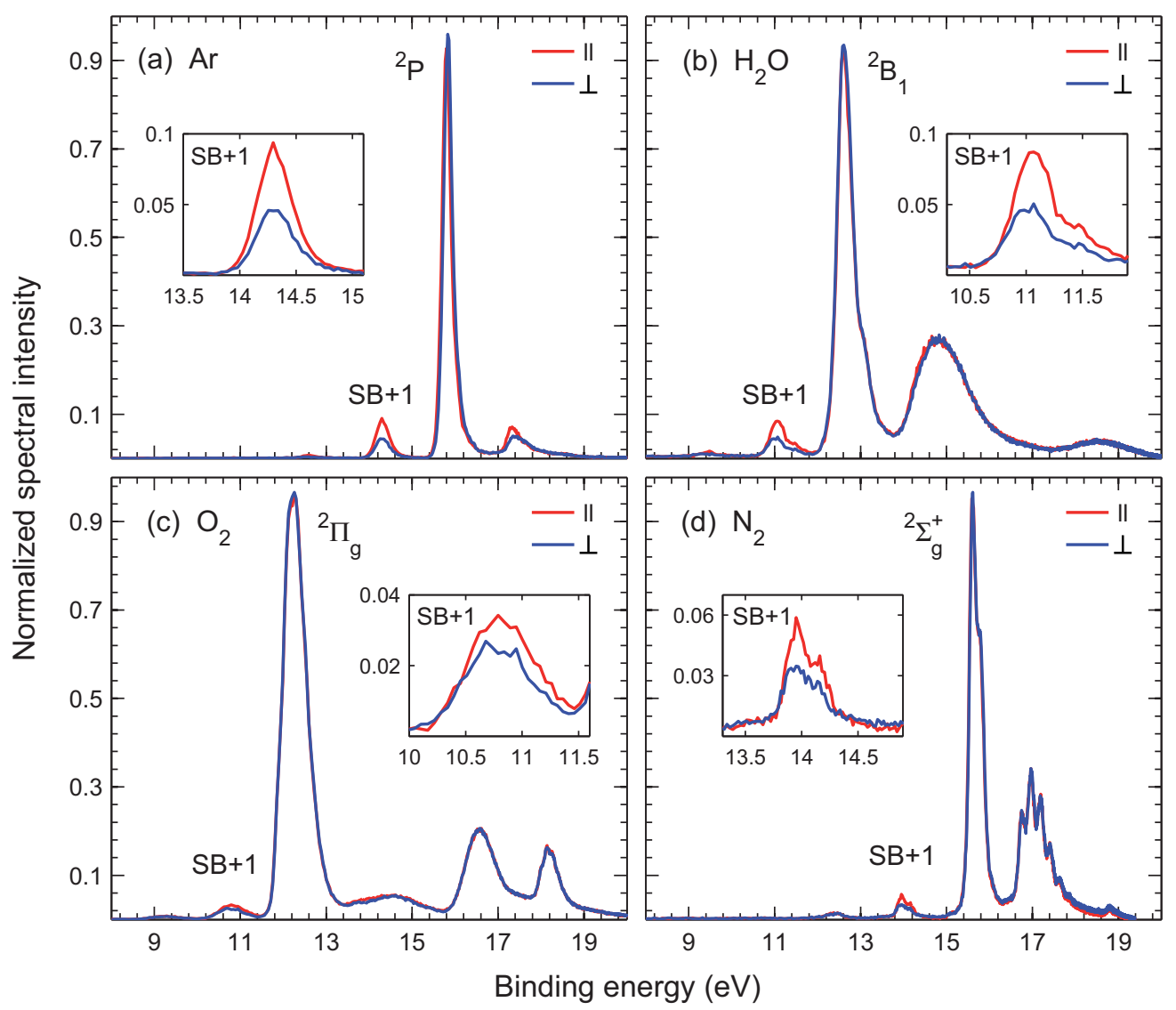

FIG. 2. (Color online) Valence photoelectron spectra of (a) Ar, (b) $\mathrm{H}_{2} \mathrm{O}$, (c) $\mathrm{O}_{2}$, and (d) $\mathrm{N}_{2}$ at temporal overlap of dressing IR (1.58 eV) and VUV (15th harmonic, $23.7 \mathrm{eV}$ ) pulses (time delay $0 \mathrm{fs}$ ) for parallel (red) and perpendicular (blue) orientations of the respective polarizations. The main lines are labeled according to the respective final ionic states. The sidebands at lower binding energies of the main lines are labeled with SB and an enhanced view of these sidebands is presented in the insets. All spectra are normalized to 1 at maximum.

these intensity modulations, the integrated sideband intensities are plotted versus the angle $\theta$ between the IR and VUV polarization vectors as depicted in Fig. 3.

The data in Fig. 3 clearly demonstrate that the amplitude of the sideband-intensity modulation with varying relative IR and VUV polarizations differs drastically for the molecules compared to the atomic case and it obviously depends on the molecule. Based on the idea that these differences arise from the different angular distributions of photoelectrons from HOMO ionization and as a first approximation to a theoretical description of the observed effects, the atomic model from Ref. [18] as developed for polarization-controlled TCATI from atoms is applied to the molecular case here. We note that we checked this model to be valid independently of the symmetry of the initial orbital. Within this model that includes the soft-photon approximation [18], the sideband intensity $I_{\mathrm{SB}}(\theta)$ varies with $\theta$ according to

$$
I_{\mathrm{SB}}(\theta) \propto 1-\frac{3 \beta_{2}}{5+2 \beta_{2}} \sin ^{2} \theta,
$$

where $\beta_{2}$ is the well-known anisotropy parameter describing the angular distribution of photoelectrons for one-photon ionization of an unpolarized, isotropic gaseous target [35]:

$$
I(\theta) \propto \frac{1}{2}\left[1+\beta_{2} P_{2}(\cos \theta)\right],
$$

with the photoelectron intensity $I(\theta)$ and the second-order Legendre polynomial $P_{2}$. In order to quantify the amplitude of the sideband-intensity modulation and following definitions in [18] the modulation amplitude $\Delta$ is introduced [see also Fig. 3(a) for a graphical definition]:

$$
\Delta=\frac{I_{\mathrm{SB}}^{\max }-I_{\mathrm{SB}}^{\min }}{I_{\mathrm{SB}}^{\max }},
$$

where $I_{\mathrm{SB}}^{\max }=I_{\mathrm{SB}}\left(0^{\circ}\right)$ and $I_{\mathrm{SB}}^{\min }=I_{\mathrm{SB}}\left(90^{\circ}\right)$ denote the maximum and minimum sideband intensities, respectively. The relationship between the experimental observable $\Delta$ and the anisotropy parameter $\beta_{2}$ then simply reads as

$$
\beta_{2}=\frac{5 \Delta}{3-2 \Delta} .
$$

Hence, with Eq. (1) it follows directly,

$$
\frac{I_{\mathrm{SB}}(\theta)}{I_{\mathrm{SB}}^{\max }}=1-\Delta \sin ^{2}(\theta) .
$$

This expression was used for the fitting of our measured data in Fig. 3 (solid red lines) in order to extract the modulation amplitudes, which are summarized in Table II. Note that the analysis does not depend on the angular momentum of the ionized electron and, as the molecules are not aligned, only the one-photon VUV photoelectron angular distribution as characterized by the beta parameter is needed. 


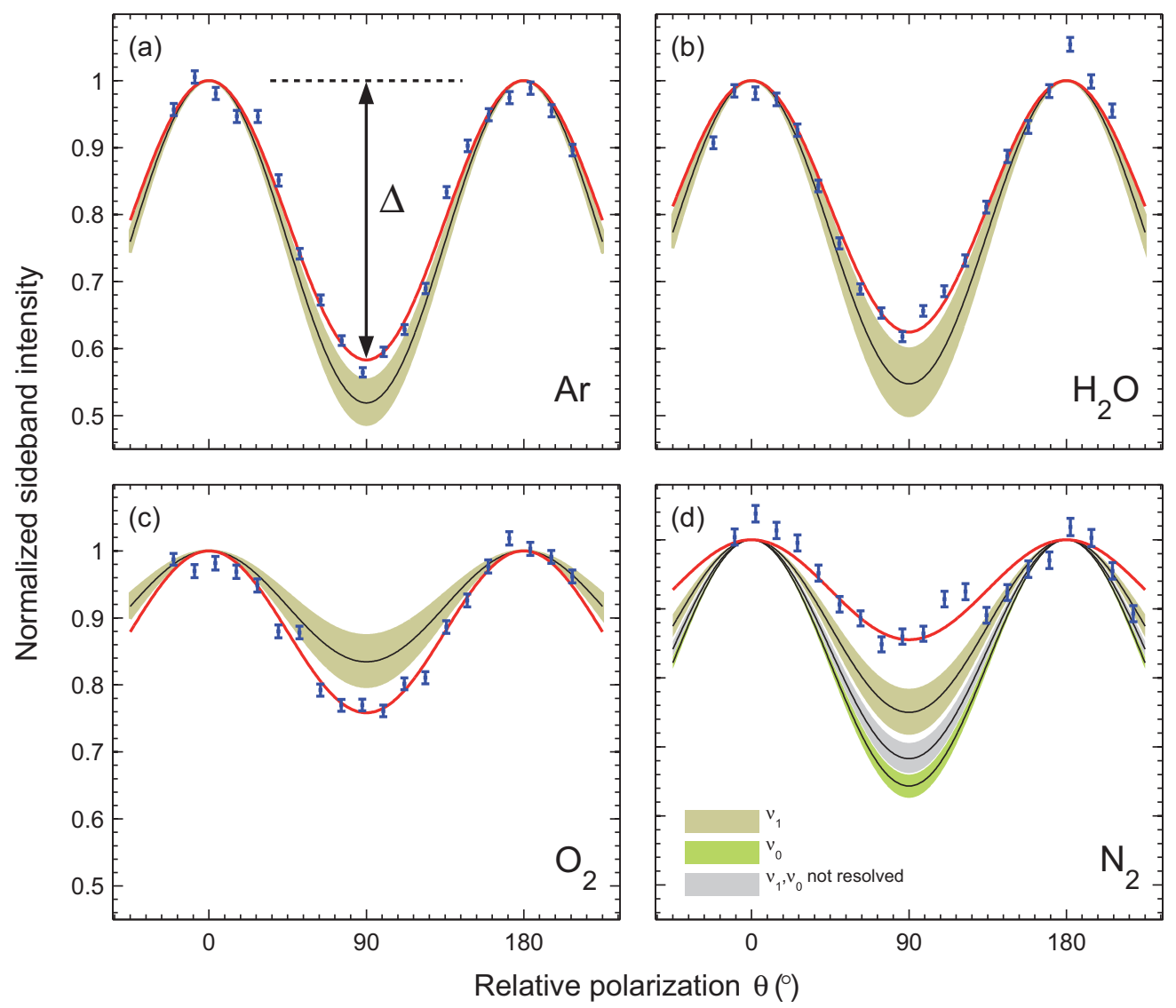

FIG. 3. (Color online) Intensities of sidebands from two-photon two-color above-threshold ionization of (a) $\mathrm{Ar}$, (b) $\mathrm{H}_{2} \mathrm{O}$, (c) $\mathrm{O}_{2}$, and (d) $\mathrm{N}_{2}$ ("SB+1" in Figs. 1 and 2) versus the angle $\theta$ between the IR and VUV polarization vectors. The data points are integrated sideband intensities and the red solid lines are fits to the measured data. Measured data are normalized such that the maxima of the fit curves are 1 . The error bars were calculated before normalization as the square root of the total electron counts and scaled with the same normalization factor as the measured data. The solid black lines depict the expected modulation based on an atomic model (see text) and previously published anisotropy parameters $\beta_{2}$ from one-photon VUV ionization at, within $0.1 \mathrm{eV}$, the same photon energy. The shaded area indicates the uncertainty interval due to the uncertainty of the reported value for $\beta_{2}$. Three expectations are given for $\mathrm{N}_{2}$ (see text). The parameter $\Delta$ introduced in (a) is used to quantify the modulation amplitude.

Apparently, $\Delta$ varies by a factor of 3 between $\mathrm{Ar}$ and $\mathrm{N}_{2}$ and by 2.5 when comparing the extreme molecular cases $\mathrm{H}_{2} \mathrm{O}$ and $\mathrm{N}_{2}$, convincingly indicating that

the polarization dependence of TCATI is a sensitive probe for the photoelectron angular distribution from molecules.

TABLE II. Fitted modulation amplitudes $\Delta$ [Eq. (3) and Fig. 3(a)] and correspondingly extracted anisotropy parameters $\beta_{2}$ [Eq. (4)] within the atomic model. The different columns refer to $3 p$ photoionization of Ar and photoionization of the highest occupied molecular orbitals $1 b_{1}$ of $\mathrm{H}_{2} \mathrm{O}, \pi_{g}^{*}$ of $\mathrm{O}_{2}$, and $\sigma_{g}$ of $\mathrm{N}_{2}$. The errors given for the experimental $\Delta$ and $\beta_{2}$ values represent the uncertainty of the model fit to the measured data (mean \pm one standard deviation). All data in this work were measured at a photon energy of $23.7 \mathrm{eV}$. Literature values for $\beta_{2}$ are given with the respective photon energies at which data were measured and the references. See text for details on $\mathrm{N}_{2}$.

\begin{tabular}{|c|c|c|c|c|c|c|}
\hline & & $\mathrm{Ar}$ & $\mathrm{H}_{2} \mathrm{O}$ & $\mathrm{O}_{2}$ & $\mathrm{~N}_{2}$ & \\
\hline \multirow[t]{2}{*}{ This work } & $\Delta$ & $0.42 \pm 0.02$ & $0.35 \pm 0.05$ & $0.24 \pm 0.03$ & $0.14 \pm 0.04$ & All measured at $23.7 \mathrm{eV}$ \\
\hline & $\rightarrow \beta_{2}$ & $0.97 \pm 0.07$ & $0.76 \pm 0.14$ & $0.48 \pm 0.07$ & $0.26 \pm 0.08$ & \\
\hline \multirow[t]{3}{*}{ Literature } & $\beta_{2}$ & $\begin{array}{c}1.18 \pm 0.12 \\
(23.62 \mathrm{eV} \\
\text { Ref. [39]) }\end{array}$ & $\begin{array}{c}1.08 \pm 0.17 \\
(23.6 \mathrm{eV} \\
\text { Ref. [50]) }\end{array}$ & $\begin{array}{c}0.31 \pm 0.08 \\
(23.8 \mathrm{eV}, \\
\text { Ref. [36] })\end{array}$ & $\begin{array}{c}0.78 \pm 0.05 \\
(23.64 \mathrm{eV}, \\
\text { Ref. [37]) }\end{array}$ & Vibrational channel $v_{0}$ \\
\hline & & & & & $\begin{array}{c}0.50 \pm 0.07 \\
(23.64 \mathrm{eV} \\
\text { Ref. [37]) }\end{array}$ & Vibrational channel $v_{1}$ \\
\hline & & & & & $\begin{array}{c}0.67 \pm 0.07 \\
(24.0 \mathrm{eV}, \\
\text { Ref. [38]) }\end{array}$ & No distinction of $v_{0}, v_{1}$ \\
\hline
\end{tabular}


In order to further test the concept, the anisotropy parameters $\beta_{2}$ were calculated from the fitted values of $\Delta$ within the atomic model with Eq. (4) (Table II). As anticipated, the sideband-intensity modulation is strongest for the most oriented electronic state with highest $\beta_{2}$. The $\beta_{2}$ values as determined here for $\mathrm{H}_{2} \mathrm{O}$ and $\mathrm{O}_{2}$ are, within the respective uncertainty intervals, in close agreement with previously published values determined from one-photon VUV ionization at the same photon energy (within $0.1 \mathrm{eV}$ ) and with angular resolution in the detection of the photoelectrons. This is surprising given the crude approximations in the applied atomic model. A graphical representation of these comparisons is made in Fig. 3 with the solid black lines representing the expected sideband-intensity modulation according to the atomic model with Eq. (1) and the literature values for $\beta_{2}$ from Table II. The curves obtained from the model deviate from the measured data by $20 \%-30 \%$. However, the model completely fails for $\mathrm{N}_{2}$.

The disagreement already observed for Ar points to a systematic uncertainty in our approach. Possibly, this is due to the neglect of higher-order sideband interactions. This speculation is supported by the very small photoelectron intensities at energies where the second sideband is expected [at $12.6 \mathrm{eV}$ in Fig. 2(a)]. The disagreement for $\mathrm{H}_{2} \mathrm{O}$ and $\mathrm{O}_{2}$ is of the same order and could thus be related to the same origin. However, whereas in $\mathrm{Ar}$ and $\mathrm{H}_{2} \mathrm{O}$ the model overestimates the modulation amplitude, it underestimates the amplitude in $\mathrm{O}_{2}$. We thus cannot exclude the influence of other effects such as the orbital delocalization in molecules compared to atoms; variations of the orbital symmetry in the initial, intermediate, and final states; and the possible influence of the molecular symmetry (noncentrosymmetry) and possible related scattering effects in angularly resolved photoionization. Note that the comparison to the literature is done for the same photon energy (within $0.1 \mathrm{eV}$ ). The bandwidth of the VUV radiation used here $(\sim 0.15 \mathrm{eV}$, [Ref. 10]) together with the comparably small variation of the $\beta_{2}$ values in this range rule out that deviations beyond the uncertainties reported in Table II are due to variations of $\beta_{2}$ with photon energy.

The disagreement for $\mathrm{N}_{2}$ is too large to be solely due to these effects, but two alternative explanations for the observed behavior can be proposed.

First, we note that in $\mathrm{N}_{2}$ the binding energy difference of electrons from the HOMO and HOMO-1 orbitals is 1.4 $\mathrm{eV}$ and thus very close to the energy of one IR photon $(1.58 \mathrm{eV})$. In $\mathrm{H}_{2} \mathrm{O}$ and $\mathrm{O}_{2}$ these energies amount to 2.1 and $4.3 \mathrm{eV}$, respectively. Only in $\mathrm{N}_{2}$ can we therefore expect a considerable contribution of three-photon TCATI by one VUV and two IR photons as depicted in Fig. 1(c). The intensities close to the binding energies of $14 \mathrm{eV}$ in the $\mathrm{N}_{2}$ spectrum, where the modulation with varying mutual VUV and IR polarizations was measured, could thus originate from the combined ionization of HOMO with one VUV and one IR photon $\left[(\mathrm{SB}+1)^{\mathrm{HOMO}}\right.$ in Fig. 1(c)] and of HOMO-1 with one VUV and two IR photons [(SB+2) ${ }^{\mathrm{HOMO}-1}$ in Fig. 1(c)]. The energies of these two channels differ by only $0.2 \mathrm{eV}$, a difference which we did not resolve in our measurement [see the inset in Fig. 2(d)]. The interference of these channels is not included in our model and could thus explain the discrepancy between the measured and modeled intensity modulation in Fig. 3 for $\mathrm{N}_{2}$. For $\mathrm{H}_{2} \mathrm{O}$ and $\mathrm{O}_{2}$ the energies for these channels would differ by 0.5 and $2.7 \mathrm{eV}$, hence reducing the possibility of this interference.

Second, we cannot exclude the influence of interfering VUV ionization channels. For $\mathrm{N}_{2}$ at $23.7 \mathrm{eV}$ the interference of direct photoionization with autoionization has been reported [37]. This leads to various vibrational final ionic states of $N_{2}^{+}$with differing $\beta_{2}$ values. Although none of these values is close to the value determined here (Table II), this interference could still explain the discrepancy between the measured and modeled anisotropy parameter as it was not included in our model. Measuring the dependence of the sideband electron angular distribution on the time delay between VUV and IR pulses was recently demonstrated to be sensitive to the relative importance of resonant and nonresonant ionization pathways [29] and could be one way to test this hypothesis.

In conclusion, we measured and simulated polarizationcontrolled two-photon two-color above-threshold ionization spectra from valence shells of molecules. The sidebandintensity modulations arising from varying the relative orientations of femtosecond infrared and vacuum-ultraviolet radiation in two-photon two-color above-threshold ionization of the highest occupied molecular orbitals of $\mathrm{H}_{2} \mathrm{O}, \mathrm{O}_{2}$, and $\mathrm{N}_{2}$ differ strongly from $3 p$ ionization of the atomic reference case $\mathrm{Ar}$. These modulations are also found to depend on the particular molecule. Modeling the modulation amplitudes with an atomic model in a first approximation allows for extracting the onephoton-ionization anisotropy parameter $\beta_{2}$. This represents an alternative way of determining photoelectron angular distributions in molecules. Deficiencies of the atomic model and in particular the large deviations between experiment and model for $\mathrm{N}_{2}$ are discussed. The large variation of the experimental observable clearly motivates using polarizationcontrolled two-photon two-color above-threshold ionization of molecules as a sensitive test of corresponding theories. Our results motivate future studies of the influence of the dressingfield density and the concomitant behavior of high-order sidebands. Furthermore, the influence of interfering channels for both the dressing IR and the VUV or x-ray radiation seem a valuable extension of the present work. Finally, an extension of polarization-controlled two-photon two-color above-threshold ionization to the core levels of molecules appears promising.

This project is supported by French state funds managed by the ANR programme ANR-09-BLAN-0031-01ATTO-WAVE, ANR Investissements d'Avenir 0031-01ATTO-WAVE, and ANR Investissements d'Avenir. M.M. acknowledges support by the Deutsche Forschungsgemeinschaft (DFG) under Grant No. SFB 925/A1.
[1] J. M. Schins, P. Breger, P. Agostini, R. C. Constantinescu, H. G. Muller, G. Grillon, A. Antonetti, and
A. Mysyrowicz, Phys. Rev. Lett. 73, 2180 (1994). 
[2] T. E. Glover, R. W. Schoenlein, A. H. Chin, and C. V. Shank, Phys. Rev. Lett. 76, 2468 (1996).

[3] T. Brabec and F. Krausz, Rev. Mod. Phys. 72, 545 (2000).

[4] P. Agostini and L. F. DiMauro, Rep. Prog. Phys. 67, 813 (2004).

[5] A. Bouhal, R. Evans, G. Grillon, A. Mysyrowicz, P. Berger, P. Agostini, R. C. Constantinescu, H. G. Muller, and D. von der Linde, J. Opt. Soc. Am. B 14, 950 (1997).

[6] E. S. Toma, H. G. Muller, P. M. Paul, P. Berger, M. Cheret, P. Agostini, C. Le Blanc, G. Mullot, and G. Cheriaux, Phys. Rev. A 62, 061801(R) (2000).

[7] J. Norin, J. Mauritsson, A. Johansson, M. K. Raarup, S. Buil, A. Persson, O. Dühr, M. B. Gaarde, K. J. Schafer, U. Keller, C.-G. Wahlström, and A. L'Huillier, Phys. Rev. Lett. 88, 193901 (2002).

[8] M. Ito, Y. Kataoka, T. Okamoto, M. Yamashita, and T. Sekikawa, Opt. Express 18, 6071 (2010).

[9] Ph. Wernet, M. Odelius, K. Godehusen, J. Gaudin, O. Schwarzkopf, and W. Eberhardt, Phys. Rev. Lett. 103, 013001 (2009).

[10] Ph. Wernet, J. Gaudin, K. Godehusen, O. Schwarzkopf, and W. Eberhardt, Rev. Sci. Instrum. 82, 063114 (2011).

[11] L. Miaja-Avila, C. Lei, M. Aeschlimann, J. L. Gland, M. M. Murnane, H. C. Kapteyn, and G. Saathoff, Phys. Rev. Lett. 97, 113604 (2006).

[12] G. Saathoff, L. Miaja-Avila, M. Aeschlimann, M. M. Murnane, and H. C. Kapteyn, Phys. Rev. A 77, 022903 (2008).

[13] M. Meyer, D. Cubaynes, P. O'Keeffe, H. Luna, P. Yeates, E. T. Kennedy, J. T. Costello, P. Orr, R. Taïeb, A. Maquet, S. Düsterer, P. Radcliffe, H. Redlin, A. Azima, E. Plönjes, and J. Feldhaus, Phys. Rev. A 74, 011401(R) (2006).

[14] S. Düsterer, P. Radcliffe, C. Bostedt, J. Bozek, A. L. Cavalieri, R. Coffee, J. T. Costello, D. Cubaynes, L. F. DiMauro, Y. Ding, G. Doumy, F. Grüner, W. Helml, W. Schweinberger, R. Kienberger, A. R. Maier, M. Messerschmidt, V. Richardson, C. Roedig, T. Tschentscher et al., New J. Phys. 13, 093024 (2011).

[15] M. Fushitani, A. Matsuda, and A. Hishikawa, J. Electron Spectrosc. Relat. Phenom. 184, 561 (2012).

[16] B. Frietsch, R. Carley, K. Döbrich, C. Gahl, M. Teichmann, O. Schwarzkopf, P. Wernet, and M. Weinelt, Rev. Sci. Instrum. 84, 075106 (2013).

[17] J. Metje, M. Borgwardt, A. Moguilevski, A. Kothe, N. Engel, M. Wilke, R. Al-Obaidi, D. Tolksdorf, A. Firsov, M. Brzhezinskaya, A. Erko, I. Yu. Kiyan, and E. F. Aziz, Opt. Express 22, 10747 (2014).

[18] P. O’Keeffe, R. López-Martens, J. Mauritsson, A. Johansson, A. L'Huillier, V. Véniard, R. Taïeb, A. Maquet, and M. Meyer, Phys. Rev. A 69, 051401(R) (2004).

[19] O. Guyétand, M. Gisselbrecht, A. Huetz, P. Agostini, R. Taïeb, V. Véniard, A. Maquet, L. Antonucci, O. Boyko, C. Valentin, and D. Douillet, J. Phys. B: At., Mol. Opt. Phys. 38, L357 (2005).

[20] M. Meyer, D. Cubaynes, D. Glijer, J. Dardis, P. Hayden, P. Hough, V. Richardson, E. T. Kennedy, J. T. Costello, P. Radcliffe, S. Düsterer, A. Azima, W. B. Li, H. Redlin, J. Feldhaus, R. Taïeb, A. Maquet, A. N. Grum-Grzhimailo, E.V. Gryzlova, and S. I. Strakhova, Phys. Rev. Lett. 101, 193002 (2008).

[21] S. J. Smith and G. Leuchs, Adv. Atom. Mol. Phys. 24, 157 (1987).
[22] K. L. Ishikawa, A. K. Kazansky, N. M. Kabachnik, and K. Ueda, Phys. Rev. A 90, 023408 (2014).

[23] O. Guyétand, M. Gisselbrecht, A. Huetz, P. Agostini, R. Taïeb, A. Maquet, B. Carré, P. Breger, O. Gobert, D. Garzella, J.-F. Hergott, O. Tcherbakoff, H. Merdji, M. Bougeard, H. Rottke, M. Böttcher, Z. Ansari, and P. Antoine, J. Phys. B: At., Mol. Opt. Phys. 41, 051002 (2008).

[24] L. H. Haber, B. Doughty, and S. R. Leone, Phys. Rev. A 79, 031401(R) (2009).

[25] L. H. Haber, B. Doughty, and S. R. Leone, J. Phys. Chem. A 113, 13152 (2009).

[26] L. H. Haber, B. Doughty, and S. R. Leone, Phys. Rev. A 84, 013416 (2011).

[27] P. O’Keeffe, A. Mihelic, P. Bolognesi, M. Zitnik, A. Moise, R. Richter, and L. Avaldi, New J. Phys. 15, 013023 (2013).

[28] T. Mazza et al., Nat. Commun. 5, 3648 (2014).

[29] S. Mondal et al., Phys. Rev. A 89, 013415 (2014).

[30] A. K. Kazansky, A.V. Grigorieva, and N. M. Kabachnik, Phys. Rev. Lett. 107, 253002 (2011).

[31] A. K. Kazansky, A.V. Grigorieva, and N. M. Kabachnik, Phys. Rev. A. 85, 053409 (2012).

[32] A. N. Grum-Grzhimailo and E. V. Gryzlova, Phys. Rev. A 89, 043424 (2014).

[33] V. Richardson, W. B. Li, T. J. Kelly, J. T. Costello, L. A. A. Nikolopoulos, S. Düsterer, D. Cubaynes, and M. Meyer, J. Phys. B: At., Mol. Opt. Phys. 45, 085601 (2012).

[34] VUV and Soft X-Ray Photoionization, edited by U. Becker and D. A. Shirley (Plenum, New York, 1996).

[35] K. Reid, Annu. Rev. Phys. Chem. 54, 397 (2003).

[36] D. G. McCoy, J. M. Morton, and G. V. Marr, J. Phys. B 11, L547 (1978).

[37] T. A. Carlson, M. O. Krause, D. Mehaffy, J. W. Taylor, F. A. Grimm, and J. D. Allen, J. Chem. Phys. 73, 6056 (1980).

[38] R. M. Holmes and G. V. Marr, J. Phys. B 13, 945 (1980)

[39] D. P. M. Holland, A. C. Parr, D. L. Ederer, J. L. Dehmer, and J. B. West, Nucl. Instrum. Methods 195, 331 (1982).

[40] L. Holmegaard, J. L. Hansen, L. Kalhøj, S. L. Kragh, H. Stapelfeldt, F. Filsinger, J. Küpper, G. Meijer, D. Dimitrovski, M. Abu-samha, C. P. J. Martiny, and L. B. Madsen, Nat. Phys. 6, 428 (2010).

[41] Ph. Wernet, J. Schulz, B. Sonntag, K. Godehusen, P. Zimmermann, A. N. Grum-Grzhimailo, N. M. Kabachnik, and M. Martins, Phys. Rev. A 64, 042707 (2001).

[42] Ph. Wernet, J. Schulz, B. Sonntag, K. Godehusen, P. Zimmermann, M. Martins, C. Bethke, and F. U. Hillebrecht, Phys. Rev. B 62, 14331 (2000).

[43] K. Godehusen, P. Zimmermann, A. Verweyen, A. von dem Borne, P. Wernet, and B. Sonntag, Phys. Rev. A 58, R3371 (1998).

[44] O. Plotzke, G. Prümper, B. Zimmermann, U. Becker, and H. Kleinpoppen, Phys. Rev. Lett. 77, 2642 (1996).

[45] A. Rouzée, F. Kelkensberg, W. K. Siu, G. Gademann, R. R. Lucchese, and M. J. J. Vrakking, J. Phys. B: At., Mol. Opt. Phys. 45, 074016 (2012).

[46] R. Guillemin, E. Shigemasa, K. Le Guen, D. Ceolin, C. Miron, N. Leclercq, K. Ueda, P. Morin, and M. Simon, Rev. Sci. Instrum. 71, 4387 (2000).

[47] R. Guillemin, O. Hemmers, D. W. Lindle, E. Shigemasa, K. Le Guen, D. Ceolin, C. Miron, N. Leclercq, P. Morin, M. Simon, and P. W. Langhoff, Phys. Rev. Lett. 89, 033002 (2002). 
[48] F. P. Sturm, M. Schöffler, S. Lee, T. Osipov, N. Neumann, H.-K. Kim, S. Kirschner, B. Rudek, J. B. Williams, J. D. Daughhetee, C. L. Cocke, K. Ueda, A. L. Landers, Th. Weber, M. H. Prior, A. Belkacem, and R. Dörner, Phys. Rev. A 80, 032506 (2009).
[49] K. Kimura, S. Katsumata, Y. Achiba, and S. Iwata, Handbook of HeI Photoelectron Spectra of Fundamental Organic Molecules (Japan Scientific Societies Press, Tokyo, 1981).

[50] D. Kilcoyne, S. Nordholm, and N. Hush, Chem. Phys. 107, 213 (1986). 